\title{
Carnets
}

Revue électronique d'études françaises de l'APEF

Deuxième série - 9 | 2017

Reconnaissances et légitimité en français

\section{Discours et légitimation}

L'initiative « Raconter la vie » de Pierre Rosanvallon

Nieves Ibeas Vuelta et Antonio Gaspar galán

\section{OpenEdition}

Journals

Édition électronique

URL : http://journals.openedition.org/carnets/2063

DOI : $10.4000 /$ carnets.2063

ISSN : 1646-7698

Éditeur

APEF

\section{Référence électronique}

Nieves Ibeas Vuelta et Antonio Gaspar galán, « Discours et légitimation », Carnets [En ligne], Deuxième série - 9 | 2017, mis en ligne le 31 janvier 2017, consulté le 30 avril 2019. URL : http://

journals.openedition.org/carnets/2063; DOI : 10.4000/carnets.2063

Ce document a été généré automatiquement le 30 avril 2019

\section{(c) (i) (8)}

Carnets est mis à disposition selon les termes de la licence Creative Commons - Atribution - Pas d'utilisation commerciale 4.0 International. 


\title{
Discours et légitimation
}

\author{
L'initiative « Raconter la vie » de Pierre Rosanvallon
}

Nieves Ibeas Vuelta et Antonio Gaspar galán

Pierre Rosanvallon est un animateur culturel à l'origine de plusieurs initiatives sociales dont Raconter la vie. En 2002, il fonde La République des Idées, projet conçu comme un « lieu de production et d'échange d'idées neuves en Europe et dans le monde. Un lien entre les personnalités, les organisations, les publications qui défendent la force des idées comme moteur de l'activité humaine $»^{1}$. Quelques années plus tard, il présente un nouveau projet - La Vie des Idées - "coopérative intellectuelle, lieu de débat et atelier du savoir » qui cherche la diffusion la plus large possible des savoirs français et internationaux et entend «tirer le meilleur parti des possibilités offertes par le web $\|^{2}$. La primauté des idées comme moteur social l'importance du débat intellectuel et l'élargissement de la diffusion pour arriver à une réflexion autant générale que possible ${ }^{3}$, ont été les caractéristiques communes de ces deux projets conçus avant la crise mondiale, financière d'abord, politique et sociale ensuite, qui a éclaté en 2008.

2 Sa nouvelle proposition Raconter la vie est encore un projet participatif qui voit le jour en 2013 pour contribuer «à rendre plus lisible la société d'aujourd'hui et à aider les individus qui la composent à s'insérer dans une histoire collective ». La diffusion des savoirs cède sa place au témoignage vital, au récit des problèmes qui angoissent les Français.e.s en tant qu'individus et en tant que société, en vue d'atteindre la cohésion sociale à travers la mise en commun. Le projet intègre, d'une part, une édition numérique de récits de vie, et de l'autre, une collection de livres publiés par Le Seuil, dont l'un des volumes, Le Parlement des invisibles, écrit par Rosanvallon lui-même, fait office de présentation de l'initiative.

3 L'édition numérique, composée de textes téléchargeables, instaure essentiellement un espace d'échange et de témoignage: des citoyen.e.s anonymes racontent leurs expériences aidé.e.s par une équipe de collaborateurs bénévoles qui mettent à point les récits envoyés ${ }^{4}$. Depuis le début du projet en janvier 2014 et jusqu'en octobre 2015, plus de 350 auteurs s'y sont exprimés. Le débat suscité par chaque récit est ouvert à la participation générale au moyen de commentaires en ligne déposés dans cet espace virtuel qui s'élargit sur internet à travers des réseaux sociaux à la recherche de lecteurs 
et lectrices de différentes conditions: facebook (https://www.facebook.com/ raconterlavie/), twitter (https://twitter.com/raconterlavie) et tumblr (http:// raconterlavie.tumblr.com/).

4 Quant aux volumes, publiés par Le Seuil sous la direction de Pauline Peretz, ils sont assurés par des auteur.e.s invité.e.s et offrent une perspective complémentaire par rapport aux récits numériques téléchargeables: d'un côté, les auteurs sont des écrivain.e.s, des professeur.e.s universitaires, des journalistes, etc., qui possèdent une certaine expérience éditoriale; de l'autre, leurs publications renvoient à des réflexions et/ou des histoires qui ont pour but de montrer, de faire voir une réalité sociale ${ }^{5}$.

\section{Légitimation sociale et légitimation discursive}

5 Le langage définit le contenu du projet, établit le cadre de communication avec les lecteurs et constitue l'espace commun où tout se développe. Les domaines littéraire et social fonctionnent comme deux champs indépendants, autonomes, possèdent leurs propres règles, mais cela n'empêche les effets de réfraction (Bourdieu, $1980: 219)$ ou les effets de prisme qui se produisent, véritables « systèmes de relations entre la littérature et les autres praxis sociales» (Viala, 1988: 64). L'écrivain.e est en fait « une instance multiple (...) qui se nourrit du caractère radicalement problématique de sa propre appartenance au champ littéraire et à la société » (Maingueneau, 2006 : §11).

En tant que responsable du projet, Rosanvallon construit un discours adressé à l'ensemble de la société française qui renvoie à un univers traversé par l'idéologie. Interlocuteurs, langage et monde social référentiel instaurent une relation que l'on peut envisager sous la forme d'un pacte de lecture, - « un pacte de lecture (...) est un Pacte sociabilité " (Viala, $1988: 68$ ) -, d'un contrat de communication (Charaudeau : 1983).

7 C'est dans cette condition singulière que nous trouvons les tentatives explicites de légitimation du projet Raconter la vie, l'enjeu de légitimation étant une des stratégies du sujet argumentant qui, d'après Charaudeau (1998: 11), vise à déterminer la position d'autorité institutionnelle et personnelle du sujet :

L'enjeu de légitimation est donc tourné vers le sujet parlant lui-même (il est tourné vers le "je") et peut se fonder sur deux types de position: (a) d'autorité institutionnelle, position qui est fondée par le statut du sujet lui conférant autorité de savoir (expert, savant, spécialiste) ou de pouvoir de décision (responsable d'une organisation); (b) d'autorité personnelle, position qui est fondée sur l'activité de persuasion et de séduction du sujet qui lui donne une autorité de fait, laquelle peut d'ailleurs se superposer à la précédente.

8 Le projet de Rosanvallon trouve sa raison d'exister dans la réalité sociale, en même temps qu'il veut être solution à la réalité problématique dans laquelle il s'inscrit. La situation sociale légitime ainsi le discours et le discours se légitime lui-même par sa capacité déclarée à chercher une issue aux conflits sociaux.

9 Le sociologue se propose de « restaurer la société dans sa dignité » et de "refonder la démocratie », entreprise qualifiée de "citoyenne », par le chemin de la « solidarité », la " redistribution » et la "réciprocité ». Conscient que la langue sert à construire la réalité et à insérer ou à exclure l'individu d'une identité collective (Klinkenberg, 2015 : 38), Rosanvallon proclame la nécessité de confronter expériences, de construire des valeurs collectives, d'intégrer au lieu d'exclure. Il se veut le porte-parole de la République et son 
projet constitue un appel au rassemblement qui a pour but de refonder la société à partir d'une perspective politique, mais aussi sociale et morale :

L'objectif de Raconter la vie est lui aussi double. Il est de faire sortir de l'ombre des existences et des lieux. Mais il est aussi de contribuer à la formation de nouvelles catégories pour appréhender la société d'aujourd'hui et en comprendre plus efficacement les ressorts et les problèmes. Il y a dans ce cadre une triple dimension : politique, sociale et morale. (Rosanvallon, $2014: 22$ )

Le succès de l'initiative est un facteur de légitimation lié à la réception de l'œuvre et aux effets sociaux subséquents, qui n'est pas encore évaluable. D'ailleurs, il serait relativement simple à déterminer le succès éditorial, mais il reste impossible à mesurer à court terme le succès social.

Du point de vue discursif, le projet est construit en vue de cette légitimation: «Toute pratique, la littéraire et l'artistique en particulier, s'assortit d'un discours d'escorte destiné à la nantir de marques de légitimité ». (Saint-Jacques et Viala, 1994 : 397). En ce sens, Raconter la vie est un discours hétérogène qui instaure une scène d'énonciation (Maingueneau, 1993) en rapport avec la communion sociale (citoyens auteurs/lecteurs) et avec l'identification vitale des Français.e.s dans les récits de vie de leurs compatriotes et avec l'existence d'une tâche collective à accomplir. L'objectif déclaré : surmonter les difficultés et atteindre la société idéale où tout individu sera parfaitement intégré. Sous la forme d'une collection de récits de vie et d'histoires "réelles ", cette scène d'énonciation présente une scénographie proche de l'agora grecque, en tant qu'assemblée de citoyens symbolique, un espace participatif où les sujets d'écriture et lecture, les thème(s), les textes, le type de publication et le contexte contribuent à fonder le processus de légitimation du projet.

\section{Légitimation du projet par l'ethos des auteur.e.s.}

Depuis la publication de la Rhétorique d'Aristote, on sait bien que l'ethos du locuteur est un atout essentiel de la valeur persuasive d'un discours. Les études à propos de la rhétorique et l'argumentation établissent un ethos construit par le discours, un ethos préalable au discours (Amossy, 1999) et même un ethos collectif, à caractère culturel (KerbratOrecchioni, $2002: 43)$.

Le projet intègre des auteur.e.s reconnu.e.s dans leurs domaines, mais aussi des auteurs presque inconnus ou même anonymes qui s'occupent des récits numériques. Dans ce casci, le sujet d'interlocution peut facilement inférer un ethos favorable à partir de l'expérience racontée, qui le renvoie, en tant que sujet-lecteur, à une situation partagée (chômage, maladie, déménagement, etc.), ${ }^{6}$ qui fait partie de sa propre existence. Par conséquent, la légitimation dans l'édition virtuelle est assurée par l'identité sociale des auteur.e.s et celle des protagonistes des histoires racontées (réalité quotidienne vécue par des citoyen.ne.s lambda).

Pour la collection publiée chez Le Seuil, le procédé est bien différent. Rosanvallon se présente comme sociologue, professeur du Collège de France, auteur de nombreuses publications concernant la démocratie et la société française, chef de groupe de recherche, directeur de plusieurs collections bibliographiques, animateur d'initiatives socioculturelles, entre autres. Les auteur.e.s qui participent de cette aventure partagent les présupposés idéologiques qui définissent l'entreprise et sont présenté.e.s en contexte, 
à l'aide d'informations concernant leur profession (poste de travail) et leur compétence (publications) ${ }^{7}$.

L'intervention de l'écrivaine Annie Ernaux dans le projet est doublement significative. D'une part, elle occupe une position légitimée dans le champ littéraire de sorte que deux phrases suffisent pour l'introduire: "Annie Ernaux est écrivain. Elle est notamment l'auteur de "La place" (1984), "La honte" (1997), "Les années" (2008) aux éditions Gallimard. (Crédit photo : Olivier Rollet) ». De l'autre, l'écriture a toujours été pour elle une façon d' « intervenir dans le monde pour le changer, si peut que ce soit », qui « n'est pas affaire de choses à dire, de "sujets" - quoique ça en fasse partie bien entendu, choisir d'écrire sur les passagers du RER n'a pas le même sens qu'écrire sur les promeneurs du jardin du Luxembourg. C'est une question de forme » (Ernaux, 2014 : 108). Ses prises de position par rapport au monde, à la politique et à l'écriture avaient déjà servi à Rosanvallon à exemplifier le modèle d'écrivain socialement engagé qu'il voulait montrer :

Cette préoccupation de faire sortir le monde social de l'invisibilité s'est à nouveau exprimée dans la période récente. Dans la littérature française, avec l'œuvre exemplaire d'Annie Ernaux qui a révélé l'intérêt du public pour une écriture qui réussissait à révéler la vie des plus humbles dans toutes ses dimensions, autant sociales que psychologiques. (Rosanvallon, $2014: 47$ )

16 Annie Ernaux illustre ainsi le paradigme bourdieusien : elle peut profiter de sa place « littéraire » et manifester un compromis politique dans le champ social (Bourdieu : 1992, 322), et elle le fait suivant un processus qu'elle considère naturel : « Écrire est, selon moi, une activité politique, c'est-à-dire qui peut contribuer au dévoiement et au changement du monde ou au contraire conforter l'ordre social, moral, existant » (Ernaux, 2003 : 74). Sa participation dans le projet est dans la nature de sa conception de l'écriture et de la littéraire.

17 L'auteure a gagné son prestige, sa légitimité en tant qu'écrivaine, dans le champ où elle est inscrite, ce qui lui permet d'avoir le « privilège » de l'engagement « ailleurs » (Dubois, 2010 : 6). Cela met en relief sa volonté d'agir et d'assumer un risque qui pourrait nuire à sa position privilégiée dans le champ littéraire, puisqu'elle doit affronter un habitus différent, des forces et des tensions nouvelles. Sa trajectoire d'engagement ailleurs que dans le champ littéraire apporte également un plus de légitimité au projet Raconter la vie. En effet, la topique de la cohérence, caractéristique du parcours professionnel de l'écrivaine, contribue sans doute à renforcer sa propre légitimité en même temps que la légitimité du projet. C'est dans la cohérence de sa trajectoire (ethos préalable) qu'il faut chercher la légitimité qu'elle apporte au projet animé par Rosanvallon.

\section{Légitimation par les textes}

Parmi les livres publiés, la collection Raconter la vie inclut un volume paru en janvier 2014, signé par Rosanvallon et intitulé Le Parlement des invisibles, qui sert de présentation/ justification/légitimation de son initiative. Ce volume décrit les problèmes de la société française, de la situation démocratique actuelle au moyen d'un lexique émouvant et émotif: "état inquiétant» de la société, "déchirements décisifs", "dérive démocratique ", «impression d'abandon " qui "exaspère et déprime les Français", "Français exclus » de la société, « démocratie minée », " frustration », etc. Un lexique de grands mots, un problème général (le malaise social français et européen que la situation 
de crise a accru) et une référence à un fait concret qui éveille des fantasmes de l'histoire de France et bouleverse une grande partie de la société : «l'inédite progression du Front National ».

À partir de cette constatation de la réalité, se manifestent des objectifs positifs d'une noblesse et d'une dignité incontestables. "Le texte contribue à produire un imaginaire social, à offrir aux groupes sociaux des figures d'identité (d'identification), à fixer des représentations du monde qui ont une fonction sociale ", écrivent Angenot et Robin (1985: 53). Le parlement des invisibles, texte qui résume le sens de toute la collection (numérique et en papier) Raconter la vie, s'autolégitime, s'appuie sur la référence de la société française pour se construire et se proclame voie de solution pour les problèmes dont elle souffre.

Or, de ce point de vue, le discours fait aussi partie de la nature du problème, du moment qu'il existe un parler faux, une langue des intentions et un parler ancré sur le monologue, tous les trois portant préjudice aux relations sociales:

La bataille pour le parler vrai se livre sur trois terrains. Sur celui du mensonge, c'est le plus évident. Mais également sur celui de la paralysie des débats liée au règne du monologue, ainsi que sur celui des problèmes posés par l'avènement d'une nouvelle langue des intentions. (Rosanvallon, 2015 : 345)

21 Les problèmes sociaux, ainsi que les possibles solutions, font partie essentielle du langage humain et de l'usage que la société actuelle en fait. Dans une certaine mesure, Rosanvallon reprend l'idée de Sartre (1948: 281) pour qui «la fonction d'un écrivain est d'appeler un chat un chat. Si les mots sont malades, c'est à nous de les guérir. Au lieu de cela, beaucoup vivent de cette maladie ». Ces déviations du langage exercent des influences réelles sur la vie des citoyen.ne.s, ${ }^{8}$ dont les conséquences sont terribles du point de vue communautaire en ce sens qu'ils « sont de la sorte cantonnés à une situation de citoyens passifs » (Rosanvallon, 2015 : 349) et cessent de participer dans la société. En effet, l'abstention grandissante aux successives élections ou la montée du Front National, constituent autant d'exemples de cette lassitude et préoccupent la plupart des intellectuels républicains. Rosanvallon voit dans cette dérive démocratique une conséquence directe de la déviation langagière :

L'invisibilité a aussi un coût démocratique. Elle laisse en effet le champ libre au développement d'un langage politique saturé d'abstractions, qui n'a plus de prise sur le réel et s'enfonce dans l'idéologie, c'est-à-dire la constitution de mondes magiques et factices. (Rosanvallon, $2014: 11$ )

Rosanvallon se sert de l'affirmation de Hannah Arendt sur la capacité des totalitarismes à couper les masses du monde réel ${ }^{9}$ pour prêcher un discours d'intégration qui rende visible les problèmes sociaux, qui permette de partager les expériences et l'identification à autrui à travers les souffrances. Cette identification, fondée sur la ressemblance et la reconnaissance sociocognitive, devrait conduire à une adhésion sociale par le biais de l'émotion. C'est ainsi que les textes de Raconter la vie pourraient contribuer «à faire reculer les idéologies de l'identité et du repli sur soi qui fondent la montée en puissance des populismes et du racisme, qui pourrissent la politique. » (Rosanvallon, $2014: 62$ )

\section{Légitimation par le pathos}

Le discours de Rosanvallon se construit sur une réalité idéologique qui est censée être partagée par une bonne partie de la société française contemporaine et qui légitime son 
projet : la politique est éloignée des problèmes quotidiens des Français, ce qui fait que les citoyens se sentent de plus en plus oubliés par l'action du gouvernement et s'intéressent de moins en moins aux discours politiques. Dans ce contexte, l'indifférence sociale envers la politique en France et en général en Europe constitue un topos incontournable.

C'est un problème de langage, mais aussi d'illisibilité, d'impossibilité de saisir les problèmes de la société actuelle et le discours y joue son rôle en établissant deux groupes. Le premier, formé par "les Français démunis", serait construit en vue de la reconnaissance des lecteurs et cherchant à éveiller leur empathie. Le deuxième, constitué par ceux « qui savent s'organiser », « les héritiers d'une tradition revendicative » ou qui " ont un accès facile aux médias » (Rosanvallon 2014 : 10).

Face à ces deux groupes émerge un «On" qui rassemblerait les représentants des institutions ignorant les difficultés qui hantent les individus sans voie de communication pour montrer leurs souffrances : les politiciens, les gouvernants, les intellectuels? Alors, si Rosanvallon se propose d'aider les gens sans ressources, de faire émerger l'iceberg social invisible aux yeux des gouvernants, comment ne pas attirer la complicité de l'interlocuteur qui, en outre, a accepté la situation discursive imposée et partage les prémisses idéologiques du discours?

Du point de vue de l'émotion, il est bien connu l'effet légitimateur des causes ressenties comme justes, comportant un caractère universel ${ }^{10}$ et représentées par un leader apprécié et reconnu par ceux qui appartiennent à la société destinataire du discours. Un leader intégrateur qui veut finir avec la frustration et la dépression des Français, qui veut guérir la société française de ses déchirements et qui compte sur tous: «Rejoignez raconterlavie.fr, le roman vrai de la société française " est écrit en quatrième de couverture des volumes de la collection, tandis que le site web témoigne aussi largement de cet objectif intégrateur : «Raconter la vie » le roman vrai de la société d'aujourd'hui. Soyez-en les personnages et les auteurs».

Ces valeurs universelles, comprises ou ressenties, contribuent à leur tour à légitimer le projet. Quand M. Espagne et M. Werner (1994: 9) définissent la littérature nationale, affirment qu'il faut chercher sa légitimité dans sa capacité à construire une histoire " conforme aux qualités présumées de la nation ». C'est précisément le cas de ce projet orienté non seulement dans le but d'offrir une réponse à l'état d'âme de la société française, mais d'apporter aussi des solutions à la désagrégation sociale et à l'individualisme dominant. Il cherche la légitimité pour représenter les sentiments des gens, des individus, de la société française, pour "insérer l'individu dans la société » et « refonder la démocratie ». "Résister et refonder» est le titre du dernier chapitre du Parlement des invisibles : Quoi de plus élevé?

\section{Légitimation du projet par la voie utilisée}

Comme nous avons signalé plus haut, le projet Raconter la vie se décline en deux formats complémentaires : une édition en papier, où des auteur.e.s expérimenté.e.s écrivent par invitation des livres d'environ 70 pages, et une édition numérique de récits courts (5-10 pages), dont les auteurs sont des gens anonymes qui veulent partager une expérience ou raconter une histoire. Les lecteurs ont la possibilité de participer via site web. ${ }^{11}$

La tâche, collective et participative, s'adresse à une société déboussolée et en crise. Les voies utilisées pour faire connaitre le projet doivent donc garder une totale cohérence 
avec le contexte social et les objectifs signalés : accès gratuit à une partie des récits de vie, possibilité pour les lecteurs et lectrices d'exprimer leurs opinions et de consulter les manifestations d'autres interlocuteurs, politique commerciale permettant d'acheter à un prix réduit (entre 5 et 7 euros) les volumes imprimés. Une édition de luxe et des livres vendus à des prix élevés feraient peu crédible un objectif participatif et de cohésion sociale et les productions de Raconter la vie respectent les codes imposés par le discours du projet: des récits téléchargeables gratuitement et des livres en format numérique et en format papier à des prix raisonnables. En fait, la dimension pragmatique du paratexte éditorial est définie par les caractéristiques de sa situation de communication et vise à influencer les lecteurs :

Pour qu'une couverture atteigne le but qu'elle s'est fixée, il est nécessaire qu'elle corresponde aux représentations attendues chez le destinataire; les pratiques éditoriales se fondent donc sur un schéma (plus ou moins clair, plus ou moins explicite) des motivations du public, de ses désirs, de ses fantasmes. (Lane, 92 : 99)

L'allure qui caractérise le projet, en cohérence avec le contexte social, contribue à renforcer le discours: les couvertures en deux couleurs (noir et rouge), la typographie usée, écaillée, aux contours irréguliers, comme oxydée par le temps... le tout sobre, chargé d'histoire, rappelle l'esthétique prolétaire des affiches des années soixante. Raconter la vie interprète la société à travers ces médiations, relie des moments historiques et se légitime même par le support matériel qui le rend possible.

\section{Légitimation par l'inscription de l'initiative dans une tradition de prestige « reconnu »}

31 Rosanvallon veut absolument montrer que son initiative n'est pas une invention toute récente. Elle constitue, bien au contraire, un autre chaînon d'une longue histoire de revendications au cœur de la société française et internationale. Plusieurs pages de Le parlement des invisibles sont consacrées à souligner les antécédents dont se réclame cette initiative : « la poésie ouvrière (38) " $^{12}$, « la littérature et l'essai » qui ont rendu visible au xIx ${ }^{e}$ siècle «la société issue de la Révolution (38)», mais aussi des romans comme «la Comédie Humaine... (41) (...) les Misérables (...) (42)». En ce qui concerne la période contemporaine, Rosanvallon cite « l'œuvre exemplaire d'Annie Ernaux » et le roman noir "sous l'impulsion d'auteurs comme Didier Daeninckx, Thierry Jonquet ou Jean-Patrick Manchette », entre autres. La longue liste inclut le journalisme d'enquête et des ouvrages collectifs dont Le Quai de Ouistreham de Florence Aubenas, La Misère du monde, publié en 1993 sous la direction de Pierre Bourdieu, ou La France invisible de 2006, qui « a pu à juste titre être considéré comme le manifeste des sociologues de la nouvelle génération ». Michel de Certeau et Michel Foucault constituent aussi deux références incontournables pour Rosanvallon.

Le panorama s'élargit dans le domaine international : l'Angleterre de Dickens, Trackeray ou Trollope, les États-Unis de Sinclair ou Steimbeck et du New Deal Federal Writers' Project, conforment la tradition «à prolonger» (47) à laquelle Raconter la vie veut adhérer. L'inscription "préoccupation » qui parcourt les siècles et les sociétés dans une tradition historique internationale semble tellement importante pour l'auteur qu'elle mérite même une annexe bibliographique spéciale à la fin du livre (« D'autres références sur l'histoire d'une préoccupation ", 67-69). Le projet se reconnaît de la sorte héritier d'une pratique littéraire nettement sociale. 


\section{Légitimation par les pairs et les institutions} légitimation. Le projet Raconter la vie a été présenté à La Charité-sur-Loire, «Ville du livre et Cité du mot », espace symbolique du point de vue de la légitimation institutionnelle. Depuis sa parution, il a fait l'objet de présentations et débats, ainsi que de comptes rendus, dont celui de Fabrice Audebrand, directeur du Pôle Formation statutaire et Coopération internationale à l'École nationale de protection judiciaire de la jeunesse, et celui de Daniel Béland de l'Université de Saskatchewan. Audebrand loue le projet et en souligne le caractère scientifique et la vocation citoyenne de créer "une communauté que chacun peut rejoindre à son gré, comme simple témoin ou comme acteur.» (2014 : 16). Quand à Béland, il signe un article paru dans la revue de la Société canadienne de Sociologie, où il qualifie l'ouvrage de «bien écrit et provocateur » avant de conclure que son succès « pourrait peut-être inspirer des initiatives similaires ailleurs dans la monde et pourquoi pas au Canada » $(2015: 104)$. l'œuvre de Rosanvallon, organisés par Sarah AlMatary et Florent Guénard, professeurs des universités de Lyon 2 et Nantes, respectivement. Une trentaine d'intellectuels y ont participé et la Lettre du Collège de France (Louis, 2015) a publié un résumé des discussions sous le titre «La démocratie en travail». Ces rencontres de Cerisy-la-Salle ont fait aussi l'objet de deux programmes de « France Culture Plus » : À voix nue et Les nouveaux chemins de la connaissance. ${ }^{13}$

Les présentations organisées par des librairies, telles que Kléber à Strasbourg, Ombre Blanche à Toulouse, Mollat à Bordeaux ou Champs Libres à Rennes, des fêtes du livre (celle de la ville de St. Etienne, entre autres) ou des recommandations de sites spécialisés, comme c'est le cas, notamment, de Initiales.org, ont apporté leur part à la diffusion du projet.

\section{Conclusion}

Le projet Raconter la vie se situe consciemment au carrefour des domaines linguistique et social pour y attaquer deux problèmes essentiels de la société française : le premier, l'illisibilité de la société actuelle, son opacité, l'isolement des individus qui la composent et qui se sentent exclus, démunis, oubliés par les discours politiques et peu ou pas concernés par le devenir collectif des Français. Le second, une utilisation fracturée de la langue que Rosanvallon qualifie de parler faux, qui isole les citoyen.ne.s, qui détruit la société du moment que le référent linguistique de ce parler faux n'est plus la réalité quotidienne vécue par les personnes, mais un monde inconnu où elles ne se voient plus représentées.

37 À partir de cette constatation, Rosanvallon conçoit un projet à dimension politique, sociale et morale, qui a pour but d'insérer les personnes dans une histoire collective et de refonder la démocratie. Il le fait par le biais de la parole et d'une collection de récits qui constituent le chemin vers la solidarité, l'intégration, la réorganisation sociale et la naissance d'un sentiment identitaire collectif. Si le langage (ou plutôt l'utilisation d'un 
langage coupé de la réalité) peut être cause d'isolement, d'individualisme, d'étrangeté, le récit peut devenir remède, intégration, sentiment collectif et appartenance au groupe.

Or, tout d'abord, Rosanvallon prône un retour à une utilisation responsable de la langue, en rapport permanent avec la réalité quotidienne, au concept de "parler vrai». Il rappelle ainsi le rapport existant entre la pratique langagière, le monde référentiel, le concept de vérité et l'honorabilité éthique des sujets qui n'ont pas cessé d'être révisés par les spécialistes depuis l'Antiquité. ${ }^{14} \mathrm{Il}$ y a dans le projet une considération sociale du langage, en même temps qu'un plaidoyer pour la langue comme élément de cohésion, de conformation de sociétés, de renforcement des liens qui rendent possible un réseau de relations humaines où toute personne peut se reconnaître comme individu appartenant à une communauté. Si le parler faux isole, le récit facilite l'intégration dans la mesure où les membres d'une communauté peuvent s'identifier avec les expériences racontées par d'autres membres de leur propre communauté.

Il y a là un discours de l'espoir, qui se veut médiateur et qui se légitime à travers une stratégie discursive où participent les auteur.e.s, les textes, les sentiments partagés des lecteurs et lectrices, la communion idéologique et les voies éditoriales utilisées. Institutions et pairs y jouent aussi leur rôle dans ce projet qui cherche à mettre sur pied une identité collective, qui se veut en quelque sorte un discours constituant ${ }^{15}$, et qui renvoie à une scénographie prophétique: un peuple perdu, sans espoir, déboussolé, accablé par un malaise général et une figure prophétique consacrée qui indique le chemin à suivre.

\section{BIBLIOGRAPHIE}

AMOSSY, Ruth (1999). «L'ethos au carrefour des disciplines : rhétorique, pragmatique, sociologie des champs ", in Ruth Amossy (dir). Images de soi dans le discours. La construction de l'ethos. Lausanne : Delachaux et Niestlé. ANGENOT, Marc et ROBIN, Régine (1985). «L'inscription du discours social dans le texte littéraire », Sociocriticism, 1, pp. 53-82.

ARISTÓTE (1998). Rhétorique, Trad. M. Dufour et A. Wartelle, Belles Lettres, Gallimard.

AUDEBRAND, Fabrice, (2014). « 'Raconter la vie': une initiative éditoriale vivifiante. », Les Cahiers Dynamiques $n^{\circ} 61$, pp. 16-17.

BÉLAND, Daniel (2015). « Book Review de l'ouvrage de Pierre Rosanvallon. Le Parlement des invisibles »Canadian Sociological Association/La Société canadienne de sociologie, 52/1, pp. 102-104.

BOURDIEU, Pierre (1980). Questions de sociologie. Paris : Minuit, « Documents ».

BOURDIEU, Pierre (1992). Les règles de l'art. Structure et genèse du champ littéraire. Paris : Seuil. CHARAUDEAU, Patrick (1998). «L'argumentation n'est peut-être pas ce que l'on croit », Le français aujourd'hui, n 123, p. 1-15. 
DUBoIS, Vincent (2010). De la « politique littéraire » à la littérature sans politique ? Des relations entre champs littéraire et politique en France. https://halshs.archives-ouvertes.fr/halshs-00498022

ERNAUX, Annie (2003). L'écriture comme un couteau. Paris : Éditions Stock.

ERNAUX, Annie (2014). Le vrai lieu. Entretiens avec Michelle Porte. Paris : Éditions Gallimard.

ESPAGNE, Michel et WERNER, Michael (1994). « Avant-propos » in Michel Espagne et Michael Werner (eds). Philologiques III. Qu'est-ce qu'une littérature nationale? Paris : Éditions de la Maison des Sciences de l'Homme, pp. 7-11.

KERBRAT-ORECCHIONI, Catherine (2002). « Système linguistique et ethos communicatif », Cahiers de praxématique, 38 , pp. 35-57.

KLINKENBERG, Jean-Marie (2015). La langue de la cité. Vivre et penser l'équité culturelle. Bruxelles : Les impressions nouvelles.

LA RÉDACTION, « Qui sommes-nous ? », La Vie des idées, 20 août 2007. ISSN: 2105-3030. URL: http://

www.laviedesidees.fr/Qui-sommes-nous.html

LANE, Philippe (1992). La périphérie du texte. Paris : Nathan.

LouIs, Marieke (2015). « La démocratie en travail », La lettre du Collège de France [En ligne], 39 | mars 2015, mis en ligne le 01 août 2015, consulté le 06 octobre 2015. URL : http://lettrecdf.revues.org/1957 MAINGUENEAU, Dominique (1993). Éléments de linguistique pour le texte littéraire. Paris : Dunod. MAINGUENEAU, Dominique (2006). «Quelques implications d'une démarche d'analyse du discours littéraire », COnTEXTES [En ligne], 1 | 2006, mis en ligne le 15 septembre 2006, consulté le 2 septembre 2015. URL : http://contextes.revues.org/93

; DOI : 10.4000/contextes.93

Rosanvallon, Pierre (2014). Le parlement des invisibles. Coll. « Raconter la vie », Paris : Seuil.

ROSANVALLON, Pierre (2015). Le bon gouvernement. Paris : Seuil.

SAINT-JACQUES, Denis et Alain VIALA (1994). « À propos du champs littéraire. Histoire, géographie, histoire littéraire », Annales Histoire, Sciences Sociales, 49, pp. 395-406.

SARTRE, Jean-Paul (1948). Qu'est-ce que la littérature ? Paris : Gallimard.

VIALA, Alain (1988). « Effets de champs, effet des prisme », Littérature, n 70, pp. 64-71.

\section{NOTES}

1. Les deux derniers, ceux d'Éric Maurin, La Fabrique du conformisme, et de Dominique Cardon, A quoi rêvent les algorithmes, sont parus respectivement en septembre et octobre 2015. Voir http:// www.repid.com/-Qui-sommes-nous-.html

2. http://www.laviedesidees.fr/Qui-sommes-nous.html?lang=fr

3. Une grande partie des articles parus dans La Vie des Idées sont traduits en anglais et présentés dans le site http://www.booksandideas.net/, afin de diffuser plus largement et dans le domaine anglophone la réflexion et les idées développées dans le site français.

4. Les derniers pour l'instant: une expérience sur le chemin de Compostelle (Didier Morisot), un distributeur de publicité qui arpente les trottoirs toulousains (Billy Bop), une femme qui quitte le 
domicile conjugal pour fuir de la violence (Cécile Cordier) ou une agression dans un foyer social (Amir Djân).

5. Pour la période citée, on compte 21 auteurs et des titres tels que Chercheur au quotidien de Sébastien Balibar (qui raconte son expérience de physicien chercheur) ou L'homme océan de Sylvie Caster (l'histoire d'un pêcheur d'Arcachon) pour ne citer que le premier et le dernier volume de cette période.

6. Ce qui constitue en fait l'objectif déclaré de Rosanvallon: tisser «les fils d'un monde commun ", « leur permettre de rassembler leur vie dans un récit qui fait sens, de s'insérer dans une histoire collective » (Rosanvallon, $2014: 23$ ).

7. À titre d'exemple, on peut lire que Sébastien Balibar est «directeur de recherche au Laboratoire de Physique Statistique de l'ENS. Membre de l'Académie des sciences, il est l'auteur de Je casse de l'eau et autres rêveries scientifiques (Le Pommier, 2008), La Pomme et l'atome (Odile Jacob 2005), et Demain la Physique (Odile Jacob, 2004 avec Edouard Brézin) ». La méthode se répète pour presque tous les auteurs de la collection imprimée.

8. Communication et gouvernance sont deux aspects différents du même malaise social: «Rendre le langage de nos institutions plus limpide ne saurait constituer la seule réponse à la méfiance qui s'est installée entre le citoyen et l'État qui le représente (...) Car la communication, comme la gouvernance, ce n'est pas simplement un problème technique. Ce qui est prétendument technique renvoie à un modèle social.» (Klinkenberg, 2015 : 290). Rosanvallon reprend à nouveau cette idée dans Le bon gouvernement $(2015: 340):$ "La démocratie est le régime qui implique une discussion permanente sur ses concepts et son vocabulaire ».

9. Rosanvallon (2015 : 334) renvoie à H. Arendt. Les origines du totalitarisme (1951).

10. «Les plus grandes vertus sont nécessairement celles qui ont le plus d'utilité pour les autres, puisque la vertu est une puissance capable d'accomplir de bonnes actions. C'est pour cela que l'on honore par-dessus tous les justes et les braves » (Aristote, 1998 : I, IX)

11. En octobre 2015, il y avait environ 3800 individus enregistrés sur le site, 3900 enregistrés sur Facebook, et quelque 1900 sur twitter, qui contribuent par leurs commentaires à enrichir le projet.

12. «On a peine à imaginer aujourd'hui l'immense audience des poètes ouvriers de l'époque. Le cordonnier Lapointe, le serrurier Gilland, le maçon Poncy, le tisserand Magu et quelques dizaines d'autres ont écrit des chansons et des poèmes fredonnés et lus avec enthousiasme aux quatre coins du pays ». (Rosanvallon, $2014: 36$ )

13. «France Culture» a consacré plusieurs émissions au projet de Rosanvallon, les toutes premières pendant le mois de décembre 2013, quelques semaines avant la parution des premiers volumes de la collection.

14. Préoccupation qui traverse disciplines et époques et dont M. Heidegger J. L. Austin, G. Frege, J. Searle, G. Steiner ou J. Habermas entre autres se sont occupés de nos jours à plusieurs reprises. 15. «Il y a dans toute société des paroles qui «font autorité » parce qu'elles se réclament d'une forme de transcendance qu'elles n'ont pas d'au-delà. Ces discours « constituants » sont ceux qui donnent sens aux actes de la collectivité » (Maingueneau, 2006 : §17). 


\section{RÉSUMÉS}

Raconter la vie est un projet participatif fondé par Pierre Rosanvallon en 2013, qui intègre un espace d'échange et d'édition virtuelle, ainsi qu'une collection de livres publiés chez Le Seuil. Le projet veut contribuer à aider les individus qui composent la société à s'insérer dans une histoire collective. Nous analysons dans cet article les stratégies discursives mises en œuvre en vue de la légitimation d'une écriture liée à un contexte social et politique déterminé, et qui se veut une solution aux problèmes sociaux.

Raconter la vie is an open project started by Pierre Rosanvallon in 2013 that consists of a space of exchange and online edition and a collection of books published by Seuil. One of the project's main goals is to contribute to the integration of the society in a collective history. In this paper we analyse the discursive strategies used to legitimate a project, which was born, bound to a specific political and social context and which pretends to establish itself as the answer to the society's problems.

\section{INDEX}

Mots-clés : légitimation, Ernaux (Annie), Raconter la vie, littérature sociale, discours Keywords : legitimation, Ernaux (Annie), Raconter la vie, social literature, discourse

\section{AUTEURS}

\section{NIEVES IBEAS VUELTA}

Un. de Zaragoza

nibeas[at]unizar.es

ANTONIO GASPAR GALÁN

Un. de Zaragoza

agaspar[at]@unizar.es 